\title{
A New Hybrid Intelligent Algorithm for Fuzzy Multiobjective Programming Problem Based on Credibility Theory
}

\author{
Zu-Tong Wang, ${ }^{1}$ Jian-Sheng Guo, ${ }^{1}$ Ming-Fa Zheng, ${ }^{2}$ and Ying Wang' \\ ${ }^{1}$ Equipment Management and Safety Engineering College, Air Force Engineering University, Xi'an 710051, China \\ ${ }^{2}$ School of Mathematics and Statistics, Xian Jiaotong University, Xian 710048, China \\ Correspondence should be addressed to Ming-Fa Zheng; mingfazheng@126.com
}

Received 17 June 2013; Accepted 21 February 2014; Published 25 March 2014

Academic Editor: Carsten Proppe

Copyright (c) $2014 \mathrm{Zu}$-Tong Wang et al. This is an open access article distributed under the Creative Commons Attribution License, which permits unrestricted use, distribution, and reproduction in any medium, provided the original work is properly cited.

\begin{abstract}
Based on the credibility theory, this paper is devoted to the fuzzy multiobjective programming problem. Firstly, the expectedvalue model of fuzzy multiobjective programming problem is provided based on credibility theory; then two new approaches for obtaining efficient solutions are proposed on the basis of the expected-value model, whose validity has been proven. For solving the fuzzy MOP problem efficiently, Latin hypercube sampling, fuzzy simulation, support vector machine, and artificial bee colony algorithm are integrated to build a hybrid intelligent algorithm. An application case study on availability allocation optimization problem in repairable parallel-series system design is documented. The results suggest that the proposed method has excellent consistency and efficiency in solving fuzzy multiobjective programming problem and is particularly useful for expensive systems.
\end{abstract}

\section{Introduction}

Many real-life problems have mainly been studied from the multiobjective optimization point of view; these problems require considering and optimizing multiple and conflicting objectives at the same time. The multiobjective programming (MOP) problem with deterministic parameters has received much research interest [1]. But the application of deterministic MOP methods to real-world problems often faces the difficulty that, for a particular problem considered, the parameters involved take unknown or incommensurable values at the moment of making the decision.

As pointed out by Zimmermann [2], various kinds of indeterminacy can be categorized as stochastic uncertainty and fuzziness. When the parameters involved in MOP problem are stochastic in nature, then the resulting problem is referred to as a stochastic MOP problem and can be solved through probability theory. The stochastic MOP methods have been widely used in many real-world decision making problems with the gradual perfection of probability theory, including power systems planning [3], distributed energy resources planning [4], and network design [5]. When the sample size is too small to estimate a probability distribution in real-life systems, information about these problems is often available in the form of vague descriptions; such types of indeterminacy are called fuzziness. The parameters involved in such kind of MOP problem are fuzzy variables, and the resulting problem is called a fuzzy MOP problem (FMOP). Due to its close description and representation of real world situation, the FMOP problem has been widely studied by researchers in a variety of fields, including both theory [6] and application [7]. These FMOP problem studies mentioned above are all based on the fuzzy set theory [8] and the concept of possibility measure [9] introduced by Zadeh.

However, as B. Liu and Y. K. Liu stated in [10], since the possibility measure has no self-duality, one event with possibility measure of 1 will not be necessary to happen, while one event with possibility measure of 0 will be possible to happen. Based on possibility measure, B. Liu and Y. K. Liu [10] proposed a self-dual set function, credibility measure, which can be considered as the improvement of possibility measure in the fuzzy decision system, and an axiomatic foundation based on credibility measure, called credibility theory, has been developed [11, 12]. From a measure-theoretic viewpoint, 
credibility theory provides a theoretical foundation for fuzzy programming as the role of probability theory in stochastic programming [13].

Unfortunately, although the credibility theory is much closer to the real-world FMOP problem, it has been overlooked so far. There has been only one research of FMOP problem considered based on credibility theory in previous work, that is, the primary objective function method proposed by Zheng et al. [14]. In this paper, the linear weighted method and the ideal point method are proposed to obtain Pareto efficient solutions in FMOP problem based on credibility theory. Since the expected value is widely used in real-life problems, the expected-value model of FMOP problem is proposed to convert the FMOP problem into a deterministic MOP problem, and the expected-value Pareto efficient solution in FMOP problem is defined based on credibility theory. Then the linear weighted method and the ideal point method are adopted to convert the expectedvalue model of FMOP problem into a single objective programming (SOP) problem, which proved that the optimal solutions to this SOP problem are expected-value Pareto efficient solutions to the original FMOP problem.

Given that the FMOP problems are always complex optimization problems with complicated feasible set, it is difficult to be solved by traditional methods; some hybrid intelligent algorithms using fuzzy simulation, artificial neural network (ANN), and genetic algorithm (GA) have been adopted for solving fuzzy programming problems [15]. However, since the traditional sampling method used in the fuzzy simulation needs to generate large-scale size of decision points through purely random sampling, the ANN requires large numbers of sample data to obtain the desired precision in model approximation, and the GA needs long time to obtain the optimal solution in model optimization; the computation cost of these hybrid intelligent algorithms is very time-expensive. A new powerful and efficient hybrid intelligent algorithm should be designed and applied to the FMOP problem to reduce the computation cost and improve the computation accuracy. For this purpose, a hybrid intelligent algorithm composed of Latin hypercube sampling (LHS), fuzzy simulation, support vector machine (SVM), and artificial bee colony (ABC) algorithm has been proposed to obtain the Pareto efficient solutions in FMOP problem based on credibility theory in this paper.

In the hybrid intelligent algorithm presented, it is broken into four phases, that is, sample phase, simulation phase, approximation phase, and optimization phase. In sample phase, the problem is addressed using Latin hypercube sampling introduced by McKay et al. [16], which is a very popular sampling method for use with computationally demanding models. It has been theoretically and experimentally proved that LHS is more precise and robust than traditional random sampling methods $[17,18]$. In simulation phase, the problem is addressed using fuzzy simulation method based on credibility theory to calculate the expected value of functions on the sample generated in sample phase. In approximation phase, the problem is addressed using a new and very promising regression technique developed by Vapnik et al. [19] in 1996, called support vector regression (SVR).
The excellent performances of SVR in approximation have been obtained in $[20,21]$. In optimization phase, the problem is addressed using the artificial bee colony ( $\mathrm{ABC}$ for short) algorithm proposed by Karaboga in 2005 [22], which is a metaheuristic bionic algorithm based on the intelligent foraging behavior of honey bees. It has been validated that its effectiveness and efficiency on algorithm performance are competitive to other optimization algorithms [23-25]. Since every phase in the hybrid intelligent algorithm is implanted with advanced methods, using the LHS and fuzzy simulation for model data collection, SVR for model approximation, and $\mathrm{ABC}$ algorithm for model optimization, respectively, it can reduce the computation cost and improve the computation accuracy greatly. The comparison result with traditional hybrid intelligent algorithm in a numerical example shows that this new algorithm is more precise and efficient.

The paper is organized in the following manner. In Section 2, some useful definitions and properties about credibility theory with application to FMOP problem are introduced. In Section 3, the mathematical models for a general FMOP problem are described, and the expectedvalue principle of FMOP problem is presented. Then, two solution methods are proposed to generate Pareto efficient solution of FMOP problem based on credibility theory, and relative proofs about its validity are also presented. In Section 4, a new powerful hybrid intelligent algorithm is proposed for solving the FMOP problem more efficiently, and a numerical example is presented to test its performance. In Section 5, an application case study on system availability design is provided to illustrate that the developed method in this paper is successful and efficient. Finally, a brief summary is given and some open points are stated for future research work in Section 6.

\section{Preliminaries}

In this section, some foundational concepts and properties of credibility theory are introduced, which will be used throughout this paper.

Given a universe $\Theta$, Wang [26] defined an ample field $\mathscr{P}(\Theta)$ on $\Theta$ as a class of subsets of $\Theta$ that is closed under arbitrary unions, intersections, and complement in $\Theta$, and Klir [27] introduced the possibility measure Pos on $\mathscr{P}(\Theta)$ such that

$(\operatorname{Pos} 1) \operatorname{Pos}(\varnothing)=0$, and $\operatorname{Pos}(\Theta)=1$;

(Pos 2) $\operatorname{Pos}\left(\bigcup_{i \in I} A_{i}\right)=\sup _{i \in I} \operatorname{Pos} A_{i}$, for any subclass $\left\{\left(A_{i} \mid i \in I\right)\right\}$ of $\mathscr{P}(\Theta)$, where $I$ is an arbitrary index set.

Definition 1 (see [10]). Let Pos be a possibility measure. The set function $\mathrm{Cr}$ defined by

$$
\operatorname{Cr}(A)=\frac{\left(1+\operatorname{Pos}(A)-\operatorname{Pos}\left(A^{C}\right)\right)}{2}, \quad A \in \mathscr{P}(\Theta),
$$

is called a credibility measure on $\mathscr{P}(\Theta)$, where $A^{C}=\Theta \backslash A$ is the set complement of $A$. 
It is easy to check that $\mathrm{Cr}$ is a self-dual set function in the sense that

$$
\operatorname{Cr}\{A\}+\operatorname{Cr}\left\{A^{C}\right\}=1, \quad A \in \mathscr{P}(\Theta) .
$$

The triplet $(\Theta, \mathscr{P}(\Theta), \mathrm{Cr})$ is referred to as a credibility space [11], in which a fuzzy vector is formally defined as follows.

Definition 2 (see [10]). An $n$-dimensional fuzzy vector is defined as a function from a credibility space $(\Theta, \mathscr{P}(\Theta), \mathrm{Cr})$ to the set of $n$-dimensional real number vectors. If $n=1$, then it is called a fuzzy variable.

Definition 3 (see [28]). Let $f$ be a real-valued measurable function from $R^{n}$ to $R$, and let $\xi_{i}$ be a fuzzy variable on $\left(\Theta_{i}, \mathscr{P}\left(\Theta_{i}\right)\right.$, Pos $), i=1,2, \ldots, n$; then $\xi=f\left(\xi_{1}, \xi_{2}, \ldots, \xi_{n}\right)$ defined by

$$
\begin{array}{r}
\xi\left(\theta_{1}, \theta_{2}, \ldots, \theta_{n}\right)=f\left(\xi_{1}\left(\theta_{1}\right), \xi_{2}\left(\theta_{2}\right), \ldots, \xi_{n}\left(\theta_{n}\right)\right) \\
\forall\left(\theta_{1}, \theta_{2}, \ldots, \theta_{n}\right) \in \Theta
\end{array}
$$

is called a fuzzy variable on $(\Theta, \mathscr{P}(\Theta)$, Pos), which is a tensor product of the $n$ credibility spaces $\left(\Theta_{i}, \mathscr{P}\left(\Theta_{i}\right)\right.$, Pos $), i=$ $1,2, \ldots, n$.

Definition 4 (see [10]). Let $\xi$ be a fuzzy variable; the expected value of $\xi$ is defined as

$$
E[\xi]=\int_{0}^{\infty} \operatorname{Cr}\{\xi \geq r\} d r-\int_{-\infty}^{0} \operatorname{Cr}\{\xi \leq r\} d r
$$

provided that at least one of the two integrals is finite.

\section{Solution Approaches}

3.1. Mathematical Formulation. Let us consider the FMOP problem as follows:

$$
\begin{aligned}
\min _{x \in R^{n}} \quad f(x, \xi) & \\
& =\left(f_{1}\left(x, \xi_{1}\right), f_{2}\left(x, \xi_{2}\right), \ldots, f_{p}\left(x, \xi_{n}\right)\right)
\end{aligned}
$$

subject to :

$$
\begin{aligned}
& g_{i}\left(x, \xi_{i}\right) \leq 0, \quad i=1,2, \ldots, m \\
& h_{k}\left(x, \xi_{k}\right)=0, \quad k=1,2, \ldots, l,
\end{aligned}
$$

where $x \in R^{n}$ is a vector of decision variables of the problem, the functions $f, g$, and $h$ are real-valued measurable functions, and $\xi=\left(\xi_{1}, \xi_{2}, \ldots, \xi_{n}\right)$ are fuzzy vectors whose components are continuous fuzzy variables, defined on the credibility space $(\Theta, \mathscr{P}(\Theta), \mathrm{Cr})$.

3.2. Expected-Value Model of FMOP Problem. As the expected value of fuzzy variable is widely used in real-life problems, the expected-value model of FMOP problem (5) is presented as follows:

$$
\begin{gathered}
\min _{x \in R^{n}} \quad E[f(x, \xi)] \\
=\left(E\left[f_{1}\left(x, \xi_{1}\right)\right], E\left[f_{2}\left(x, \xi_{2}\right)\right], \ldots,\right. \\
\left.E\left[f_{p}\left(x, \xi_{n}\right)\right]\right)
\end{gathered}
$$

subject to :

$$
\begin{aligned}
& E\left[g_{i}\left(x, \xi_{i}\right)\right] \leq 0, \quad i=1,2, \ldots, m \\
& E\left[h_{k}\left(x, \xi_{k}\right)\right]=0, \quad k=1,2, \ldots, l,
\end{aligned}
$$

where $E\left[f_{j}\left(x, \xi_{j}\right)\right], E\left[g_{i}\left(x, \xi_{i}\right)\right]$, and $E\left[h_{k}\left(x, \xi_{k}\right)\right]$ are the expected value of the fuzzy objectives $f_{j}\left(x, \xi_{j}\right), g_{i}\left(x, \xi_{i}\right)$, and $h_{k}\left(x, \xi_{k}\right)$, respectively.

Since the objectives in expected-value model of FMOP problem are usually in conflict, there is no optimal solution that simultaneously minimizes all objective functions. In this case, the concept of expected-value Pareto solution should be introduced, which is defined as follows.

Definition 5. A Pareto efficient solution $x^{*}$ in problem (6) is said to be expected-value Pareto efficient to the FMOP problem (5), where the feasible solution $x^{*}$ is said to be a Pareto efficient solution of problem (6) if there is no feasible solution $x$ such that

$$
E\left[f_{j}\left(x, \xi_{j}\right)\right] \leq E\left[f_{j}\left(x^{*}, \xi_{j}\right)\right], \quad j=1,2, \ldots, p,
$$

and $E\left[f_{j}\left(x, \xi_{j}\right)\right]<E\left[f_{j}\left(x^{*}, \xi_{j}\right)\right]$ for at least one index $j$.

If we denote the constraint conditions as

$$
\begin{gathered}
D=\left\{x \in R^{n} \mid E\left[g_{i}\left(x, \xi_{i}\right)\right] \leq 0, i=1,2, \ldots, m ;\right. \\
\left.E\left[h_{k}\left(x, \xi_{k}\right)\right]=0, k=1,2, \ldots, l\right\},
\end{gathered}
$$

then the expected-value model can be presented as the following equivalent model:

$$
\begin{aligned}
\min _{x \in D} E & {[f(x, \xi)] } \\
& =\left(E\left[f_{1}\left(x, \xi_{1}\right)\right], E\left[f_{2}\left(x, \xi_{2}\right)\right], \ldots, E\left[f_{p}\left(x, \xi_{n}\right)\right]\right),
\end{aligned}
$$

where the set of feasible solutions $D \subset R^{n}$ is nonempty and compact.

3.3. Linear Weighted Method. The first method we proposed is the linear weighted method, which converts the expectedvalue model in (9) into a deterministic single objective programming (SOP) problem by weighting the objective functions according to the importance of each objective; that is,

$$
\min _{x \in D} E[f(x, \xi)]=\sum_{j=1}^{p} \lambda_{j} E\left[f_{j}\left(x, \xi_{j}\right)\right],
$$

where $\lambda \in \Lambda^{++}=\left\{\lambda=\left(\lambda_{1}, \ldots, \lambda_{p}\right)^{T} \mid \lambda_{j}>0, \sum_{j=1}^{p} \lambda_{j}=1\right\}$ 
Theorem 6. The optimal solution to the deterministic SOP problem in (10) using linear weighted method must be expectedvalue Pareto efficient to the original model of FMOP problem in (5).

Proof. Suppose that $x^{*}$ is the optimal solution to the deterministic SOP problem in (10), but it is not expected-value Pareto efficient to the original model of FMOP problem (5). Following Definition 5, we can obtain that there must exist some $\bar{x}$ such that $E\left[f_{j}\left(\bar{x}, \xi_{j}\right)\right] \leq E\left[f_{j}\left(x^{*}, \xi_{j}\right)\right]$ and $E\left[f_{j}\left(\bar{x}, \xi_{j}\right)\right]<E\left[f_{j}\left(x^{*}, \xi_{j}\right)\right]$ for at least one index $j, j=$ $1,2, \ldots, p$.

Since $\lambda \in \Lambda^{++}$, we can get that

$$
\sum_{j=1}^{p} \lambda_{j} E\left[f_{j}\left(\bar{x}, \xi_{j}\right)\right]<\sum_{j=1}^{p} \lambda_{j} E\left[f_{j}\left(x^{*}, \xi_{j}\right)\right] ;
$$

that is to say, $E[f(\bar{x}, \xi)]<E\left[f\left(x^{*}, \xi\right)\right]$. In other words, $x^{*}$ is not the optimal solution to the deterministic SOP problem in (10), which contradicts with the previous hypothesis that $x^{*}$ is expected-value Pareto efficient to the original model of FMOP problem (5). The theorem is proved.

3.4. Ideal Point Method. The second method we proposed is the ideal point method, which converts the expectedvalue model in (9) into a deterministic single objective programming (SOP) problem by minimizing the distance function from a solution

$$
\left(E\left[f_{1}\left(x, \xi_{1}\right)\right], E\left[f_{2}\left(x, \xi_{2}\right)\right], \ldots, E\left[f_{p}\left(x, \xi_{p}\right)\right]\right),
$$

to an ideal vector $\left(f_{1}^{0}, f_{2}^{0}, \ldots, f_{p}^{0}\right)$, where $f_{j}^{0}$ denotes the optimal values of the $j$ th objective functions on the set of feasible solution $D$ without considering other objectives, $j=$ $1,2, \ldots, p$, respectively. That is,

$$
\min _{x \in D} E[f(x, \xi)]=\sqrt{\sum_{j=1}^{p}\left(E\left[f_{j}\left(x, \xi_{j}\right)\right]-f_{j}^{0}\right)^{2}} .
$$

Theorem 7. The optimal solution to the deterministic SOP problem in (13) using ideal point method must be expectedvalue Pareto efficient to the original model of FMOP problem in (5).

Proof. Suppose that $x^{*}$ is the optimal solution to the deterministic SOP problem in (13), but it is not expected-value Pareto efficient to the original model of FMOP problem (5). Following Definition 5, we can obtain that there must exist some $\bar{x}$ such that $E\left[f_{j}\left(\bar{x}, \xi_{j}\right)\right] \leq E\left[f_{j}\left(x^{*}, \xi_{j}\right)\right]$ and $E\left[f_{j}\left(\bar{x}, \xi_{j}\right)\right]<E\left[f_{j}\left(x^{*}, \xi_{j}\right)\right]$ for at least one index $j, j=$ $1,2, \ldots, p$.

Without any loss of generality, let us assume when $j=j_{0}$, $E\left[f_{j_{0}}\left(\bar{x}, \xi_{j_{0}}\right)\right]<E\left[f_{j_{0}}\left(x^{*}, \xi_{j_{0}}\right)\right]$. As $f_{j_{0}}^{0}$ is the optimal value of $E\left[f_{j_{0}}\left(x, \xi_{j_{0}}\right)\right]$, then

$$
\left(E\left[f_{j_{0}}\left(\bar{x}, \xi_{j_{0}}\right)\right]-f_{j_{0}}^{0}\right)^{2}<\left(E\left[f_{j_{0}}\left(x^{*}, \xi_{j_{0}}\right)\right]-f_{j_{0}}^{0}\right)^{2} .
$$

When $j \neq j_{0}$, it is obvious that

$$
E\left[f_{j}\left(\bar{x}, \xi_{j}\right)\right] \leq E\left[f_{j}\left(x^{*}, \xi_{j}\right)\right] .
$$

Since $f_{j}^{0}$ denotes the optimal value of single objective $E\left[f_{j}\left(x, \xi_{j}\right)\right]$ (for $j=1,2, \ldots, p$ ) on feasible set, then we can get that

$$
\left(E\left[f_{j}\left(\bar{x}, \xi_{j}\right)\right]-f_{j}^{0}\right)^{2} \leq\left(E\left[f_{j}\left(x^{*}, \xi_{j}\right)\right]-f_{j}^{0}\right)^{2}, \quad\left(j \neq j_{0}\right) .
$$

Then, it follows that

$$
\sum_{j=1}^{p}\left(E\left[f_{j}\left(\bar{x}, \xi_{j}\right)\right]-f_{j}^{0}\right)^{2}<\sum_{j=1}^{p}\left(E\left[f_{j}\left(x^{*}, \xi_{j}\right)\right]-f_{j}^{0}\right)^{2} .
$$

And it further gets that

$$
\sqrt{\sum_{j=1}^{p}\left(E\left[f_{j}\left(\bar{x}, \xi_{j}\right)\right]-f_{j}^{0}\right)^{2}}<\sqrt{\sum_{j=1}^{p}\left(E\left[f_{j}\left(x^{*}, \xi_{j}\right)\right]-f_{j}^{0}\right)^{2}} ;
$$

that is to say, $E[f(\bar{x}, \xi)]<E\left[f\left(x^{*}, \xi\right)\right]$. In other words, $x^{*}$ is not the optimal solution to the deterministic SOP problem in (13), which contradicts with the previous hypothesis that $x^{*}$ is expected-value Pareto efficient to the original model of FMOP problem (5). The theorem is proved.

\section{Hybrid Intelligent Algorithm for FMOP Problem}

To perform multiobjective optimization under fuzziness, the most direct approach is to nest the iterative loops by performing complete fuzzy estimation (inner loop) for each optimization data request (outer loop). However, this can be prohibitively expensive; for this reason, the optimization techniques must be combined with hybrid intelligent algorithm. In this section, the Latin hypercube sampling (LHS), fuzzy simulation, support vector regression (SVR), and artificial bee colony (ABC) algorithm are integrated to design a powerful hybrid intelligent algorithm for solving FMOP problem.

4.1. Generating Sample Using LHS. The goal of sampling is to generate a matrix of experiments $X^{n}=\left(x_{i j}\right)_{n \times k}$, where $n$ is the number of experiments and $k$ is the number of variables. As an extension of stratified-random procedure, Latin hypercube sampling has a long history and has shown its robustness capabilities in sample generation. The LHS involves sampling $n s$ values from the prescribed distribution of each of $k$ decision variables $X_{1}, X_{2}, \ldots, X_{k}$ in FMOP problem. Unlike simple random sampling, LHS ensures a full coverage of the range of each variable by maximally stratifying each marginal distribution.

As the information of design variables in FMOP problem is always hard-available beforehand, we assume that all design variables follow uniform distribution. The main steps of LHS in FMOP problem can be summarized as follows. 
Step 1: divide the cumulative distribution of each design variable into $N$ equiprobable intervals.

Step 2: select a value from each interval randomly; once a point is selected in an interval, no other point could be selected in this interval. For the ith interval, the sampled cumulative probability can be written as Prob $_{i}=(1 / N) r_{u}+(i-1) / N$, where $r_{u}$ is uniformly distributed random number ranging from 0 to 1 .

Step 3: transform the probability values sampled into the value using the inverse of the distribution function $F^{-1}: x=F^{-1}$ (Prob).

Step 4: the $N$ values obtained for each design variable $x$ are paired randomly with the $n s$ values of other design variables.

4.2. Computing the Objective Functions Using Fuzzy Simulation. Due to the complexity of fuzzy objective functions, we adopt the fuzzy simulation to calculate the expected value of objective functions on the sample generated by LHS. The main steps of fuzzy simulation in FMOP problem can be summarized as follows.

Step 1: choose one sampling point $x_{0}$ from the sample generated by LHS.

Step 2: set $e=0$.

Step 3: generate $\theta_{k}$ from $\Theta$ according to the possibility measure Pos, and assure that $\operatorname{Pos}\left(\theta_{k}\right) \geq \varepsilon, k=$ $1,2, \ldots, n$, where $n$ is a sufficiently large number and $\varepsilon$ is a sufficiently small number.

Step 4: set $a=f\left(x_{0}, \xi\left(\theta_{1}\right)\right) \wedge \cdots \wedge f\left(x_{0}, \xi\left(\theta_{n}\right)\right)$ and $b=f\left(x_{0}, \xi\left(\theta_{1}\right)\right) \vee \cdots \vee f\left(x_{0}, \xi\left(\theta_{n}\right)\right)$.

Step 5: generate $r$ from uniformly $[a, b]$.

Step 6: if $r \geq 0$, then $e \leftarrow e+\operatorname{Cr}\left\{f\left(x_{0}, \xi\right) \geq r\right\}$.

Step 7: if $r<0$, then $e \leftarrow e-\operatorname{Cr}\left\{f\left(x_{0}, \xi\right) \leq r\right\}$.

Step 8: repeat the fifth to seventh steps for $N$ times, where $N$ is a sufficiently large number.

Step 9: return $E\left[f\left(x_{0}, \xi\right)\right]=a \vee 0+b \wedge 0+e \cdot(b-a) / N$.

Step 10: return to Step 1 until all the sample points have been chosen out.

In Steps 6 and 7, there involves the calculation of credibility measure; following Definition 1 of credibility measure, we can obtain that

$$
\begin{aligned}
& \operatorname{Cr}\left\{f\left(x_{0}, \xi\right) \geq r\right\} \\
& =\frac{\left(1+\operatorname{Pos}\left\{f\left(x_{0}, \xi\right) \geq r\right\}-\operatorname{Pos}\left\{f\left(x_{0}, \xi\right)<r\right\}\right)}{2}, \\
& \operatorname{Cr}\left\{f\left(x_{0}, \xi\right) \leq r\right\} \\
& =\frac{\left(1+\operatorname{Pos}\left\{f\left(x_{0}, \xi\right) \leq r\right\}-\operatorname{Pos}\left\{f\left(x_{0}, \xi\right)>r\right\}\right)}{2} .
\end{aligned}
$$

4.3. Approximating the Expected-Value Model Using SVR. After obtaining the sample in feasible set and the corresponding expected values of objective functions, it needs to build a surrogate model to map the relationships between them, which can be considered as a regression process. Support vector regression (SVR) is a new regression method different from traditional/statistical ones; it minimizes the generalized error bound instead of the observed training error, so as to achieve the generalized performance. In this paper, we start our study on the basis of the SVM toolbox-LIBSVM directly [29], rather than discussing the principle and algorithm of SVR. LIBSVM is a library for SVM, whose goal is to let users easily use SVM as a tool. Since the control parameters of SVR are very sensitive to its performance, a successful parameter selection is very important, especially the parameter $\gamma$ in kernel function and the parameter $C$ of cost. The artificial bee colony $(\mathrm{ABC})$ algorithm is used to find the optimal control parameters aiming at the best regression accuracy, called ABC-SVR. The main steps of the ABC-SVR application in the FMOP problem can be summarized as follows.

Step 1: generate train data and test data based on the sample and its corresponding expected values of objective functions.

Step 2: normalize the train data and test data to improve the regression ability of SVR.

Step 3: denote the parameter $\gamma$ in kernel function and the parameter $C$ of cost as a food position $(c, \gamma)$ and the regression accuracy as the nectar amount.

Step 4: adopt ABC algorithm to find the optimal control parameters of SVR (optimal food position) on the normalized data.

Step 5: use the optimal control parameters to build a SVR model and train it according to the train data.

Step 6: employ the test data to validate the accuracy of trained SVR model. If the regression accuracy does not meet, return to Step 3 to change the ABC options until the desired accuracy is met.

4.4. Optimization Using ABC Algorithm. Created by Karaboga [22], the artificial bee colony algorithm is a new population-based metaheuristic method that is motivated by the intelligent foraging behaviors of honeybee swarm. In the ABC algorithm, there are three essential components, that is, food source positions, nectar amount, and three kinds of foraging bees (employed bees, onlookers, and scouts). Each food source position represents a feasible solution to the optimization problem being considered and the nectar amount of a food source corresponds to the quality (fitness) of the solution being represented by that food source. Each kind of foraging bee performs one particular operation for generating new candidate food source positions. The ABC algorithm visualizes the employed and onlooker bees as performing the job of local search (exploitation) whereas the onlookers and scouts bees as performing the job of global search (exploration). Specifically, unlike real bee colonies, the $\mathrm{ABC}$ algorithm assumes that there is a one-to-one 


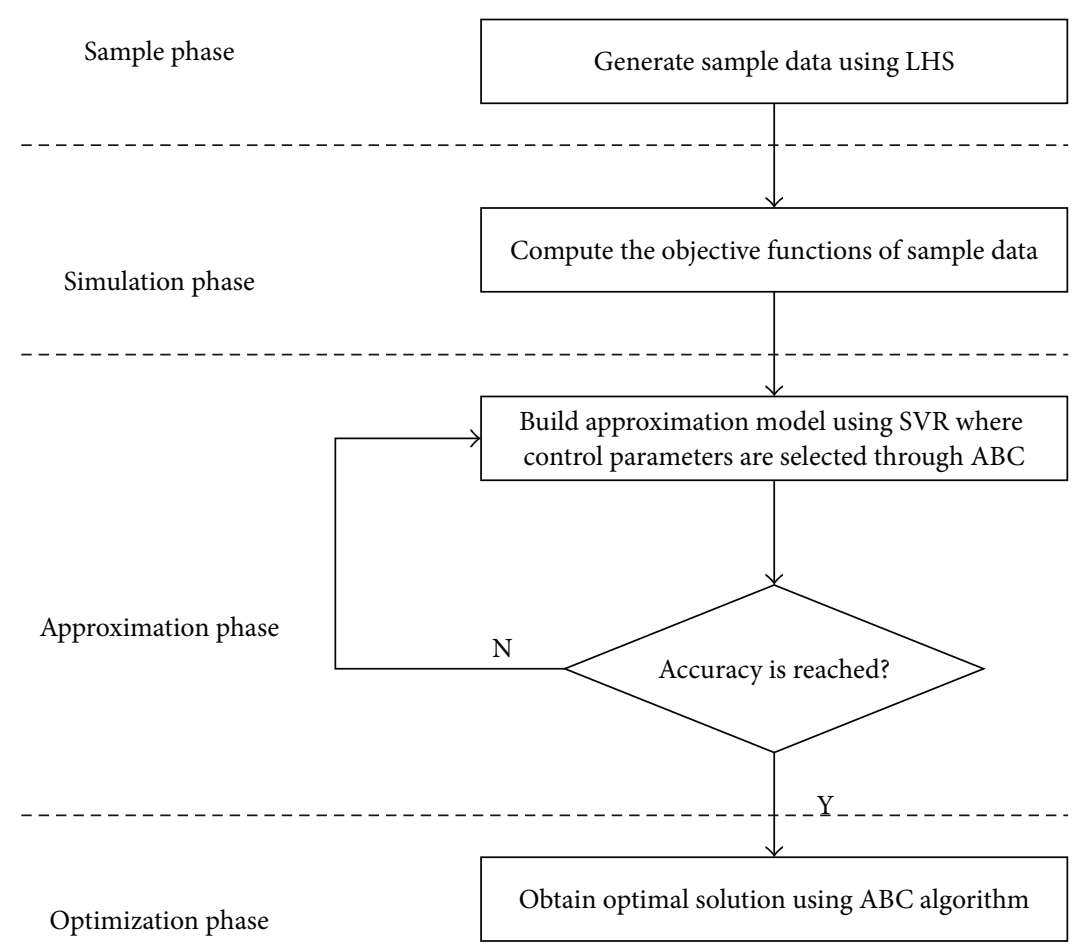

FIGURE 1: Framework of hybrid intelligent algorithm for FMOP problem.

correspondence between the employed bees and the food sources; that is, the number of food sources (solution) is the same as the number of employed bees. The main steps of $\mathrm{ABC}$ algorithm in the FMOP problem can be summarized as follows.

Step 1: initialize the control parameters, including the number of food sources or the number of the employed bees, number of onlooker bees, number of scout bees, and number of cycles.

Step 2: initialize population of food source with random solutions.

Step 3: send the employed bees to the food sources and determine the nectar amounts $f_{i}$ using the SVR model built in Section 4.3.

Step 4: calculate the fitness values of each solution fit $_{i}$ and its corresponding probability values as follows:

$$
\begin{aligned}
& \text { fit }_{i}= \begin{cases}\frac{1}{\left(1+f_{i}\right)} & \text { if } f_{i} \geq 0, \\
1+\mathrm{abs}\left(f_{i}\right) & \text { if } f_{i}<0,\end{cases} \\
& p_{i}=\frac{\text { fit }_{i}}{\sum_{i=1}^{S N} \mathrm{fit}_{i}},
\end{aligned}
$$

where $i=1,2, \ldots, S N$ and $S N$ is the number of food sources.

Step 5: send the onlooker bees to their food sources according to the probability values.
Step 6: send the scouts to the search area if a food source could not be improved through "limit" trials and replace it with a new randomly produced solution if the new solution is better.

Step 7: memorize the best food source (solution) achieved so far.

Step 8: if a stopping criterion is met, then output the best food source; otherwise, go back to Step 3.

Straightforwardly, the framework of the proposed hybrid intelligent algorithm is illustrated in Figure 1. It can be seen that the hybrid intelligent algorithm not only applies classic sample method to generate sample data in feasible set but also adopts advanced regression procedure to build surrogate model and powerful optimization algorithm for solution improvement.

4.5. Performance Comparison with the Traditional Hybrid Intelligent Algorithm. To test the performance of the proposed hybrid intelligent algorithm, it is compared with the traditional hybrid intelligent algorithm on a numerical example from [30], which is presented as follows:

$$
\begin{array}{ll}
\max & E\left[\sqrt{\left\|x_{1}-\xi_{1}\right\|+\left\|x_{2}-\xi_{2}\right\|+\left\|x_{3}-\xi_{3}\right\|}\right] \\
\text { s.t. } & x_{1}^{2}+x_{2}^{2}+x_{3}^{2} \leq 10,
\end{array}
$$

where $\xi_{1}, \xi_{2}$, and $\xi_{3}$ are triangular fuzzy variables $(-4,-2,0)$, $(-2,0,-2)$, and $(0,2,4)$, respectively. In the traditional hybrid 


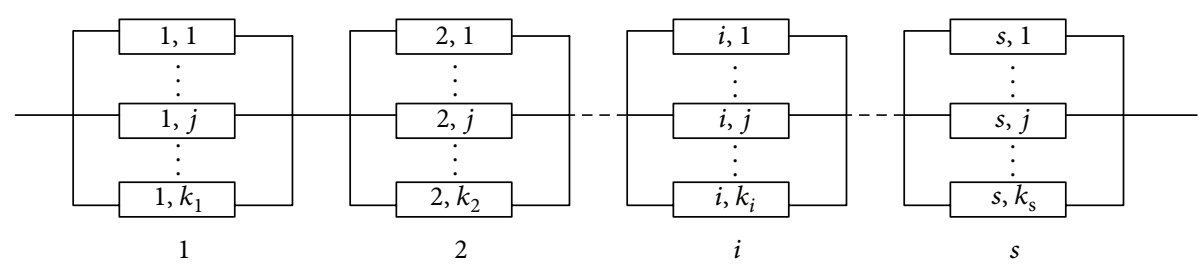

FIGURE 2: General structure of a parallel-series system.

TABle 1: Performance comparison.

\begin{tabular}{lcc}
\hline & Traditional algorithm & Proposed algorithm \\
\hline Optimal solution & $(-1.7809,-1.8632$, & $(-1.1469,-2.3775$, \\
& $-1.8322)$ & $-1.7375)$ \\
Minimum objective & 3.77 & 3.94 \\
Time cost & 1659 seconds & 185 seconds \\
\hline
\end{tabular}

intelligent algorithm, it needs to produce 2000 sample data to train the ANN and 6000 generations in the evolution of GA. While in the new algorithm, it just needs to produce 500 sample data and go through 60 cycles. The results obtained using these two algorithms are shown in Table 1. It can be obtained that the proposed algorithm is more precise and efficient than the traditional hybrid intelligent algorithm.

\section{Application Case Study}

The approach for solving FMOP problems proposed above is illustrated through an availability optimization problem in repairable parallel-series system design. A parallel-series system is a system made of parallel subsystems put in serial, which can be shown as in Figure 2. A parallel subsystem works when at least one of its components works and a series system fails when at least one of its components fails. The problem of availability optimization in system design can be formulated as a multiobjective optimization problem: minimize the cost and maximize the availability of the global system simultaneously. Uncertainty has always been an important consideration when designing and analyzing systems. It assumes that the failure rate and repair rate of components are fuzzy variables in this case, and its membership function can be available before system design using expert knowledge. In this example, we suppose that the subsystems are parallel, and that in each subsystem all components are identical. Here, the "identical" term means that the failure rate and repair rate of components have the same membership function in fuzzy environment. Then the decision variables of this problem are the number of components $k_{i}$ in each subsystem $(i=1,2, \ldots, s)$, and the availability optimization problem is to find the optimal values of $k_{i}$ to minimize the cost and maximize the system availability.

5.1. Mathematical Model of the Problem. The formulation of the mathematical model taking into account all the influential factors is very important for the success of optimization. Let
TABLE 2: Nomenclature used in the mathematical model (22).

\begin{tabular}{|c|c|}
\hline Variable & Signification \\
\hline$s$ & Number of subsystems \\
\hline$i$ & Index of a subsystem \\
\hline$\lambda_{i}$ & $\begin{array}{l}\text { Fuzzy variable of the failure rate of components in } \\
\text { subsystem } i \text { with known membership function }\end{array}$ \\
\hline$\lambda$ & $\lambda=\left(\lambda_{1}, \lambda_{2}, \ldots, \lambda_{s}\right)$ \\
\hline$\mu_{i}$ & $\begin{array}{l}\text { Fuzzy variable of the repair rate of components in } \\
\text { subsystem } i \text { with known membership function }\end{array}$ \\
\hline$\mu$ & $\mu=\left(\mu_{1}, \mu_{2}, \ldots, \mu_{s}\right)$ \\
\hline$k_{i}$ & Number of components in subsystem $i$ \\
\hline$k$ & $k=\left(k_{1}, k_{2}, \ldots, k_{s}\right)$ \\
\hline$l_{k}, u_{k}$ & $\begin{array}{l}\text { Vector of minimum and maximum number of } \\
\text { components allowable in each subsystem }\end{array}$ \\
\hline$a_{i}, b_{i}, p_{i}, q_{i}$ & $\begin{array}{l}\text { Coefficients of the cost function which can be } \\
\text { obtained from maintenance database }\end{array}$ \\
\hline
\end{tabular}

$k_{i}, A_{s}$, and $C_{s}$ denote the number of components in each subsystem, the availability of the system, and the system cost, respectively. Then the FMOP problem can be formulated as follows:

$$
\begin{array}{ll}
\max _{k} & A_{s}(\lambda, \mu, k)=\prod_{i=1}^{s}\left(1-\left(\frac{\lambda_{i}}{\lambda_{i}+\mu_{i}}\right)^{k_{i}}\right), \\
\min _{k} & C_{s}(\lambda, \mu, k)=\sum_{i=1}^{s} k_{i}\left(a_{i} \lambda_{i}^{p_{i}}+b_{i} \mu_{i}^{q_{i}}\right),
\end{array}
$$

subject to :

$$
l_{k i} \leq k_{i} \leq u_{k i} \quad(i=1,2, \ldots, s), k_{i} \in N_{+} .
$$

The variables in the FMOP model above are illustrated in Table 2 .

Since maximizing the availability $A_{s}$ is equivalent to minimizing the unavailability $\left(1-A_{s}\right)$, the mathematical model can be transformed as follows:

$$
\begin{array}{ll}
\min _{k} & U_{s}(\lambda, \mu, k)=1-A_{s}(\lambda, \mu, k) \\
\min _{k} & C_{s}(\lambda, \mu, k)
\end{array}
$$

subject to :

$$
l_{k i} \leq k_{i} \leq u_{k i} \quad(i=1,2, \ldots, s), k_{i} \in N_{+} .
$$


TABLE 3: The input data of problem.

\begin{tabular}{lc}
\hline Membership function of $\lambda_{i}$ & $10^{-3} \times(2+0.2(i-1), 2.5+0.2(i-1), 3+0.2(i-1), 3.5+0.2(i-1)), i=1,2, \ldots, 6$ \\
Membership function of $\mu_{i}$ & $(0.1+0.05(i-1), 0.15+0.05(i-1), 0.2+0.05(i-1), 0.25+0.05(i-1)), \quad i=1,2, \ldots, 6$ \\
$l_{k}$ & $1 \times(1,1,1,1,1,1)$ \\
$u_{k}$ & $6 \times(1,1,1,1,1,1)$ \\
a & $0.01 \times(4,2,8,5,12,8)$ \\
b & $0.1 \times(0.4,0.2,0.8,1,1.2,0.8)$ \\
p & $-0.8 \times(0.4,0.2,0.8,1,1.2,0.8)$ \\
q & $0.85 \times(0.4,0.2,0.8,1,1.2,0.8)$ \\
\hline
\end{tabular}

TABLE 4: The first ten sample points generated by LHS.

\begin{tabular}{lc}
\hline Sample point (SP) & Sample data \\
\hline SP1 & $(2,2,5,3,5,2)$ \\
SP2 & $(2,4,5,2,1,3)$ \\
SP3 & $(5,5,5,4,2,5)$ \\
SP4 & $(5,2,3,2,5,2)$ \\
SP5 & $(5,6,2,6,4,4)$ \\
SP6 & $(5,5,3,4,3,5)$ \\
SP7 & $(2,6,5,4,5,2)$ \\
SP8 & $(2,4,2,5,3,2)$ \\
SP9 & $(2,4,1,1,4,6)$ \\
SP10 & $(4,3,5,2,6,2)$ \\
\hline
\end{tabular}

TABLE 5: Fuzzy simulation results of the first ten sample points.

\begin{tabular}{lcc}
\hline $\begin{array}{l}\text { Sample point } \\
\text { (SP) }\end{array}$ & $\begin{array}{c}\text { The expected value of } \\
\text { unavailability } U_{s}\end{array}$ & $\begin{array}{c}\text { The expected value of } \\
\text { cost } C_{s}\end{array}$ \\
\hline SP1 & $8.7025 e-04$ & 135.7135 units \\
SP2 & 0.0169 & 57.1931 units \\
SP3 & $3.7900 e-04$ & 105.1304 units \\
SP4 & 0.0018 & 176.9077 units \\
SP5 & $8.9794 e-04$ & 187.3301 units \\
SP6 & $3.2716 e-05$ & 161.0648 units \\
SP7 & 0.0035 & 250.7263 units \\
SP8 & 0.0047 & 179.1654 units \\
SP9 & 0.0959 & 213.2756 units \\
SP10 & 0.0021 & 338.1837 units \\
\hline
\end{tabular}

5.2. Input Data of the Optimization Problem. After constructing the mathematical model, our next task is to determine the input data of the optimization problem. It is assumed that the system considered consists of six parallel subsystems in serial; that is to say, $s=6$. Since the trapezium fuzzy variables have been widely used in system reliability analysis, $\lambda_{i}, \mu_{i}$ are supposed to be trapezium fuzzy variables. The input data we assumed are shown in Table 3.

5.3. Problem Solution. In this subsection, we will try to solve the FMOP problem described above based on credibility theory using the approaches proposed in this paper.

5.3.1. Equivalent Transformation of FMOP Problem. Here, two approaches proposed in Section 3 are adopted to transform it into a deterministic SOP problem for generating
TABLE 6: Control parameters adopted in the ABC algorithm.

\begin{tabular}{lc}
\hline \multicolumn{3}{c}{ Control parameters in ABC algorithm } \\
\hline Colony size & 40 \\
Limit & 100
\end{tabular}

Number of onlookers

Number of employed bees

Half of the colony size

Number of scouts

Half of the colony size 1

Pareto efficient solutions. Using linear weighted method, it can obtain the equivalent SOP model as follows:

$$
\min _{k} \quad \omega_{1} E\left[U_{s}(\lambda, \mu, k)\right]+\omega_{2} E\left[C_{s}(\lambda, \mu, k)\right]
$$

subject to :

$$
l_{k i} \leq k_{i} \leq u_{k i} \quad(i=1,2, \ldots, s), k_{i} \in N_{+} .
$$

Using ideal point method, it can obtain the equivalent SOP model as follows:

$$
\begin{array}{ll}
\min _{k} & \sqrt{\left\|E\left[U_{s}(\lambda, \mu, k)\right]-U_{s}^{0}\right\|^{2}+\left\|E\left[C_{s}(\lambda, \mu, k)\right]-C_{s}^{0}\right\|^{2}} \\
\text { s.t. } & l_{k i} \leq k_{i} \leq u_{k i} \quad(i=1,2, \ldots, s), k_{i} \in N_{+},
\end{array}
$$

where $U_{s}^{0}$ and $C_{s}^{0}$ denote the optimal expected value of unavailability $U_{s}$ and $C_{s}$ without considering other objectives, respectively.

5.3.2. Sample Phase. As the information of the number of components in each subsystem is unknown beforehand, it is assumed that all design variables $k_{i},(i=1,2, \ldots, 6)$ follow uniform distribution $U(1,6)$. Because $k_{i}$ are discrete value, we use LHS to generate sample in the feasible set and take the integers nearest to the sample value generated by LHS. We set the sample size as 300 , and the first ten sample points are shown in Table 4.

5.3.3. Simulation Phase. We adopt the fuzzy simulation to calculate the expected value of unavailability $U_{s}\left(E\left[U_{s}\right]\right)$ and cost $C_{s}\left(E\left[C_{s}\right]\right)$ on the 300 sample points generated by LHS. We set the number of expected-value calculation cycle (outer loop) as 500 and the number of credibility measure calculation cycle (inner loop) as 5000. The simulation results of the first ten sample points are shown in Table 5. 
TABLE 7: Comparison results of three methods (ABC-SVM/SVM/ANN) about $E\left[U_{s}\right]$.

\begin{tabular}{lccr}
\hline Method & Parameters & \multicolumn{2}{c}{ Regression performance } \\
& & Train data & Test data \\
\hline ABC-SVM & best $c=1.5105$ and best $g=0.4064$ & $245 / 250(98 \%)$ & $47 / 50(94 \%)$ \\
SVM & best $c=0.7071$ and best $g=0.3536$ & $216 / 250(86.4 \%)$ & $32 / 50(64 \%)$ \\
ANN & Hidden layer number $=10$ & $187 / 250(74.8 \%)$ & $26 / 50(52 \%)$ \\
\hline
\end{tabular}

TABLE 8: Comparison results of three methods (ABC-SVM/SVM/ANN) about $E\left[C_{s}\right]$.

\begin{tabular}{lcrr}
\hline \multirow{2}{*}{ Method } & Parameters & \multicolumn{2}{c}{ Regression performance } \\
& & Train data & Test data \\
\hline ABC-SVM & best $c=3.3354$ and best $g=0.0100$ & $248 / 250(99.2 \%)$ & $48 / 50(96 \%)$ \\
SVM & best $c=22.6274$ and best $g=0.0020$ & $221 / 250(88.4 \%)$ & $34 / 50(68 \%)$ \\
ANN & Hidden layer number $=10$ & $198 / 250(79.2 \%)$ & $26 / 50(52 \%)$ \\
\hline
\end{tabular}

5.3.4. Approximation Phase. After obtaining the sample in feasible set and the corresponding expected values of objective functions, we use ABC-SVR method to build the surrogate model. Since the precision required in the calculation of $E\left[U_{s}\right]$ and $E\left[C_{s}\right]$ is very high, it is very difficult to obtain an ideal surrogate model to predict $E\left[U_{s}\right]$ and $E\left[C_{s}\right]$. However, we can use the ABC-SVR to build a surrogate model to map the order relationship between sample and objectives. In other words, even though we cannot predict the $E\left[U_{s}\right]$ and $E\left[C_{s}\right]$ of a sample point with high precision, we can predict the order of $E\left[U_{s}\right]$ and $E\left[C_{s}\right]$ in all sample objectives correctly. That is to say, the sample point with minimum objectives we predicted is also the corresponding sample point with minimum objectives in sample data; the optimal solution we get in the surrogate model is also the optimal solution in real problem. After getting the optimal solution, the fuzzy simulation is adopted to obtain the corresponding $E\left[U_{s}\right]$ and $E\left[C_{s}\right]$ with high precision. We take the 1-250 sample data as the train data and the 251300 sample data as the test data to validate the surrogate model. Two surrogate models are built to predict $E\left[U_{s}\right]$ and $E\left[C_{s}\right]$, respectively. The accuracy of order prediction is used to represent the regression performance and compare the regression performance of ABC-SVR with normal SVR using grid search method and BP artificial neural network. In the ABC algorithm, the maximum number of cycles was taken as 2500. The percentages of onlooker bees and employed bees were half of the colony and the number of scout bees was selected to be one. Parameters set for the ABC algorithm are given in Table 6 , and the comparison result is shown in Tables 7 and 8.

As shown in Tables 7 and 8 , the regression performance of $\mathrm{ABC}-\mathrm{SVM}$ is the best. Furthermore, it has achieved an ideal precision.

5.3.5. Optimization Phase. After obtaining the two surrogate models of $E\left[U_{s}\right]$ and $E\left[C_{s}\right]$, the $\mathrm{ABC}$ algorithm is adopted to find the optimal solution of deterministic SOP problem (24) (25). In the ABC algorithm, control parameters set for the $\mathrm{ABC}$ algorithm are the same as shown in Table 6.
TABLE 9: Results obtained in model (24).

\begin{tabular}{lcc}
\hline Scenarios & \multicolumn{2}{c}{ Results } \\
\hline$\omega_{1}=0.7, \omega_{2}=0.3$ & $E\left[U_{s}\right]$ & $2.7216 e-06$ \\
& $E\left[C_{s}\right]$ & 103.6331 units \\
& Optimal solution & $(5,5,4,4,3,5)$ \\
\hline \multirow{2}{*}{$\omega_{1}=0.5, \omega_{2}=0.5$} & $E\left[U_{s}\right]$ & $5.6275 e-06$ \\
& $E\left[C_{s}\right]$ & 100.0452 units \\
& Optimal solution & $(5,5,5,3,3,4)$ \\
$\omega_{1}=0.3, \omega_{2}=0.7$ & $E\left[U_{s}\right]$ & 0.0394 \\
& $E\left[C_{s}\right]$ & 35.6331 units \\
& Optimal solution & $(3,3,1,1,1,3)$
\end{tabular}

TABLE 10: Results obtained in model (25).

\begin{tabular}{lc}
\hline & Results \\
\hline$E\left[U_{s}\right]$ & $1.8425 e-04$ \\
$E\left[C_{s}\right]$ & 73.7527 units \\
Optimal solution & $(4,5,4,3,2,3)$ \\
\hline
\end{tabular}

In the solution of model (24), three scenarios are considered here; they are $\omega_{1}=0.7, \omega_{2}=0.3, \omega_{1}=0.5, \omega_{2}=0.5$, $\omega_{1}=0.3$, and $\omega_{2}=0.7$, respectively. The results obtained are shown in Table 9.

As shown in Table 9, the results obtained are different in three scenarios; this is due to the fact that different weights denote different importance of the objectives and higher weight implies higher importance. Therefore, the optimal solutions obtained are different.

Next, we consider the solution of model (25). Using ABC algorithm, we can obtain the minimum values of $E\left[U_{s}\right]$ and $E\left[C_{s}\right]$ on the feasible set without considering other objectives. They are $3.5894 \mathrm{e}-10$ and 30.412316692 , respectively. Then, we can solve the model (25) using the same way in the solution of model (24). The results obtained are shown in Table 10.

\section{Conclusion}

In this paper, two new solution approaches were proposed to generate Pareto efficient solutions for FMOP problem based 
on credibility theory, which are different from the traditional FMOP solution methods. To solve the FMOP problem efficiently based on credibility theory, Latin hypercube sampling (LHS), fuzzy simulation, support vector regression (SVR), and artificial bee colony (ABC) algorithm are integrated to design a powerful hybrid intelligent algorithm. An application case study on system availability optimization was provided to show the modeling of FMOP problem based on credibility theory and validate the performance of the hybrid intelligent algorithm. Specifically, given that the availability optimization problem requires a very high precision, we suggest finding the optimal solution according to the order of objectives, instead of the real values of objectives. And then the precise optimal values of objectives can be obtained using fuzzy simulation after we get the optimal solution.

In our view, the following problems in the FMOP field should be studied based on credibility theory in future, some of these problems are as follows.

(1) Other deterministic models of FMOP problem should be proposed and considered based on credibility theory, such as the variance model, $\alpha$ optimistic value model, $\alpha$-pessimistic value model, and credibility measure model.

(2) Since the FMOP problem is transformed into a deterministic MOP problem directly, it will neglect the fuzzy nature of original problem to some extent. A new method is needed for converting the FMOP problem into a fuzzy SOP problem first.

\section{Conflict of Interests}

The authors declare that there is no conflict of interests regarding the publication of this paper.

\section{Acknowledgments}

This work was supported by the National Natural Science Foundation under Grant no. 71171199 and the Natural Science Foundation of Shaanxi Province under Grant no. 2013JM1003.

\section{References}

[1] M. Özlen and M. Azizoğlu, "Multi-objective integer programming: a general approach for generating all non-dominated solutions," European Journal of Operational Research, vol. 199, no. 1, pp. 25-35, 2009.

[2] H.-J. Zimmermann, "Fuzzy programming and linear programming with several objective functions," Fuzzy Sets and Systems, vol. 1, no. 1, pp. 45-55, 1978.

[3] J. Teghem Jr. and P. L. Kunsch, "Application of multi-objective stochastic linear programming to power systems planning," Engineering Costs and Production Economics, vol. 9, no. 1-3, pp. 83-89, 1985.

[4] A. Alarcon-Rodriguez, G. Ault, and S. Galloway, "Multiobjective planning of distributed energy resources: a review of the state-of-the-art," Renewable and Sustainable Energy Reviews, vol. 14, no. 5, pp. 1353-1366, 2010.
[5] A. Chen, J. Kim, S. Lee, and Y. Kim, "Stochastic multi-objective models for network design problem," Expert Systems with Applications, vol. 37, no. 2, pp. 1608-1619, 2010.

[6] F. Arıkan and Z. Güngör, "A two-phase approach for multiobjective programming problems with fuzzy coefficients," Information Sciences, vol. 177, no. 23, pp. 5191-5202, 2007.

[7] D. Peidro, J. Mula, R. Poler, and J.-L. Verdegay, "Fuzzy optimization for supply chain planning under supply, demand and process uncertainties," Fuzzy Sets and Systems, vol. 160, no. 18, pp. 2640-2657, 2009.

[8] L. A. Zadeh, "Fuzzy sets," Information and Control, vol. 8, no. 3, pp. 338-353, 1965.

[9] L. A. Zadeh, "Fuzzy sets as a basis for a theory of possibility," Fuzzy Sets and Systems, vol. 100, no. 1, pp. 9-34, 1999.

[10] B. Liu and Y.-K. Liu, "Expected value of fuzzy variable and fuzzy expected value models," IEEE Transactions on Fuzzy Systems, vol. 10, no. 4, pp. 445-450, 2002.

[11] B. Liu, "A survey of credibility theory," Fuzzy Optimization and Decision Making, vol. 5, no. 4, pp. 387-408, 2006.

[12] Y.-K. Liu, "Convergent results about the use of fuzzy simulation in fuzzy optimization problems," IEEE Transactions on Fuzzy Systems, vol. 14, no. 2, pp. 295-304, 2006.

[13] B. Liu, "Toward fuzzy optimization without mathematical ambiguity," Fuzzy Optimization and Decision Making, vol. 1, no. 1, pp. 43-63, 2002.

[14] M. Zheng, B. Li, and G. Kou, "The expected value model of multiobjective programming and its solution method based on bifuzzy environment," Journal of Computers, vol. 6, no. 9, pp. 1942-1948, 2011.

[15] B. Liu and K. Iwamura, "Topological optimization models for communication network with multiple reliability goals," Computers \& Mathematics with Applications, vol. 39, no. 7-8, pp. 59-69, 2000.

[16] M. D. McKay, R. J. Beckman, and W. J. Conover, "A comparison of three methods for selecting values of input variables in the analysis of output from a computer code," Technometrics, vol. 21, no. 2, pp. 239-245, 1979.

[17] M. Stein, "Large sample properties of simulations using Latin hypercube sampling," Technometrics, vol. 29, no. 2, pp. 143-151, 1987.

[18] A. A. Giunta, J. M. McFarland, L. P. Swiler, and M. S. Eldred, "The promise and peril of uncertainty quantification using response surface approximations," Structures and Infrastructure Engineering, vol. 2, no. 3-4, pp. 175-189, 2006.

[19] V. Vapnik, S. E. Golowich, and A. Smola, "Support vector method for function approximation, regression estimation, and signal processing," in Advances in Neural Information Processing Systems, pp. 281-287, MIT Press, Cambridge, Mass, USA, 1997.

[20] H. Drucker, C. J. C. Burges, L. Kaufman, A. Smola, and V. Vapnik, "Support vector regression machines," in Advances in Neural Information Processing Systems, pp. 155-161, MIT Press, Cambridge, Mass, USA, 1997.

[21] M. Stitson, A. Gammerman, V. Vapnik, V. Vovk, C. Watkins, and J. Weston, "Support vector regression with anova decomposition kernels," in Advances in Kernel Methods-Support Vector Learning, pp. 285-292, MIT Press, Cambridge, Mass, USA, 1999.

[22] D. Karaboga, "An idea based on honey bee swarm for numerical optimization," Tech. Rep., 2005.

[23] D. Karaboga and B. Akay, "A comparative study of artificial Bee colony algorithm," Applied Mathematics and Computation, vol. 214, no. 1, pp. 108-132, 2009. 
[24] D. Karaboga and B. Basturk, "On the performance of artificial bee colony (ABC) algorithm," Applied Soft Computing Journal, vol. 8, no. 1, pp. 687-697, 2008.

[25] D. Karaboga and B. Basturk, "A powerful and efficient algorithm for numerical function optimization: artificial bee colony (ABC) algorithm," Journal of Global Optimization, vol. 39, no. 3, pp. 459-471, 2007.

[26] P. Z. Wang, "Fuzzy contactability and fuzzy variables," Fuzzy Sets and Systems, vol. 8, no. 1, pp. 81-92, 1982.

[27] G. J. Klir, "On fuzzy-set interpretation of possibility theory," Fuzzy Sets and Systems, vol. 108, no. 3, pp. 263-273, 1999.

[28] B. Liu, Uncertainty Theory: An Introduction to Its Axiomatic Foundations, vol. 154 of Studies in Fuzziness and Soft Computing, Springer, Berlin, Germany, 2004.

[29] C.-C. Chang and C.-J. Lin, "LIBSVM: a Library for support vector machines," ACM Transactions on Intelligent Systems and Technology, vol. 2, no. 3, article 27, 2011.

[30] B. Liu, R. Zhao, and G. Wang, Uncertain Programming with Application, Tsinghua University, 5th edition, 2012, (Chinese). 


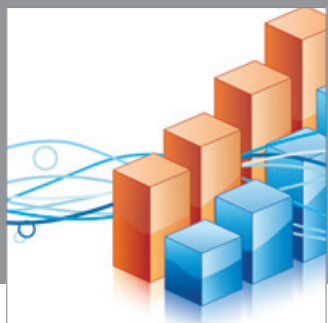

Advances in

Operations Research

mansans

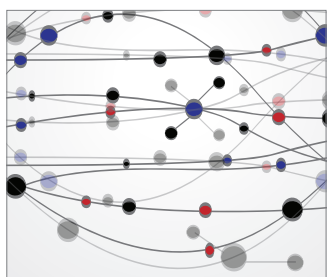

The Scientific World Journal
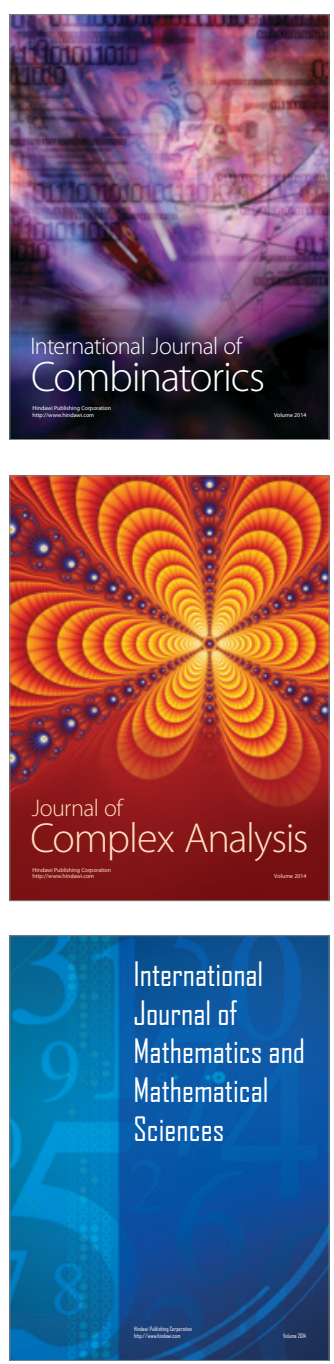
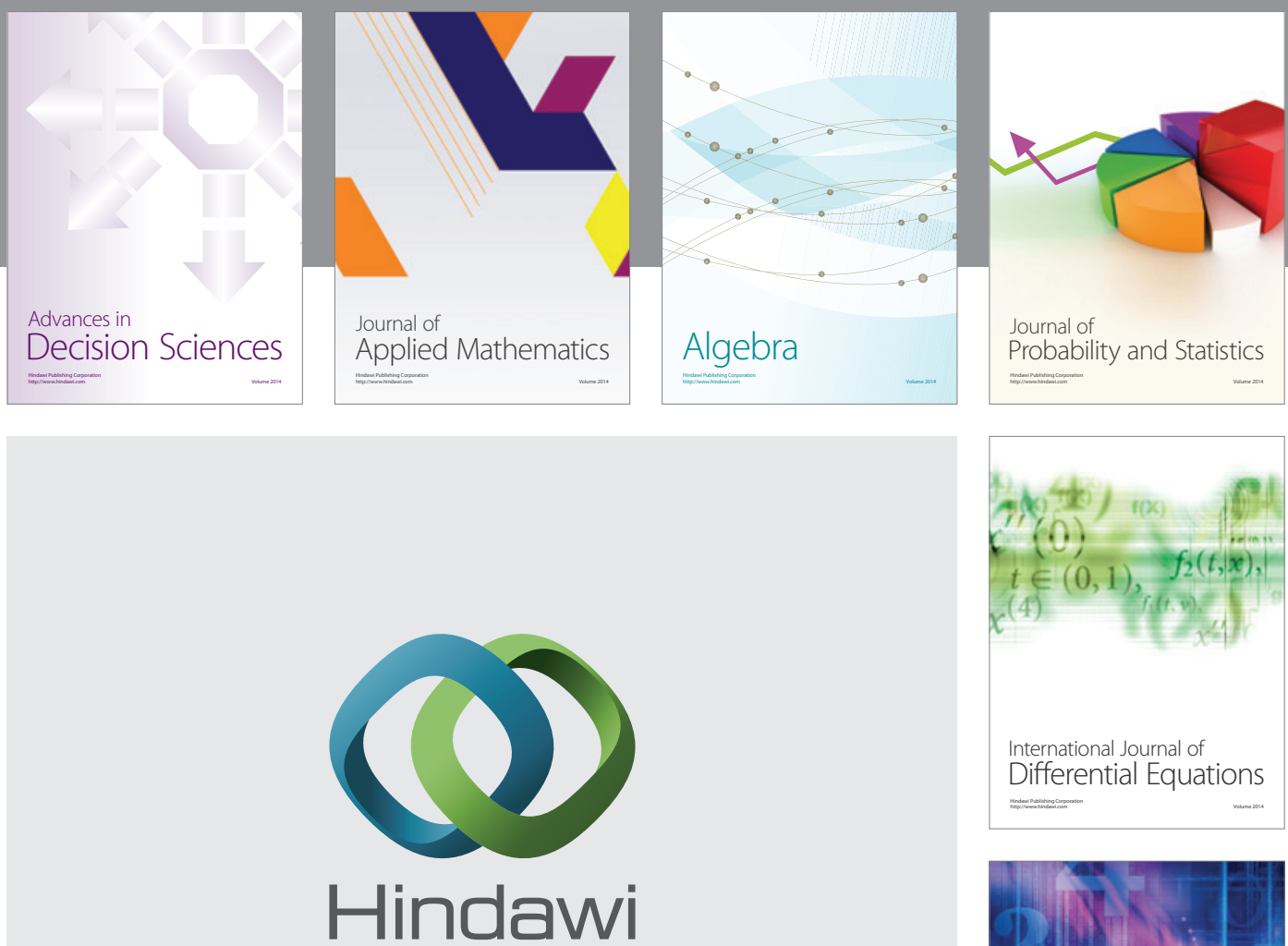

Submit your manuscripts at http://www.hindawi.com
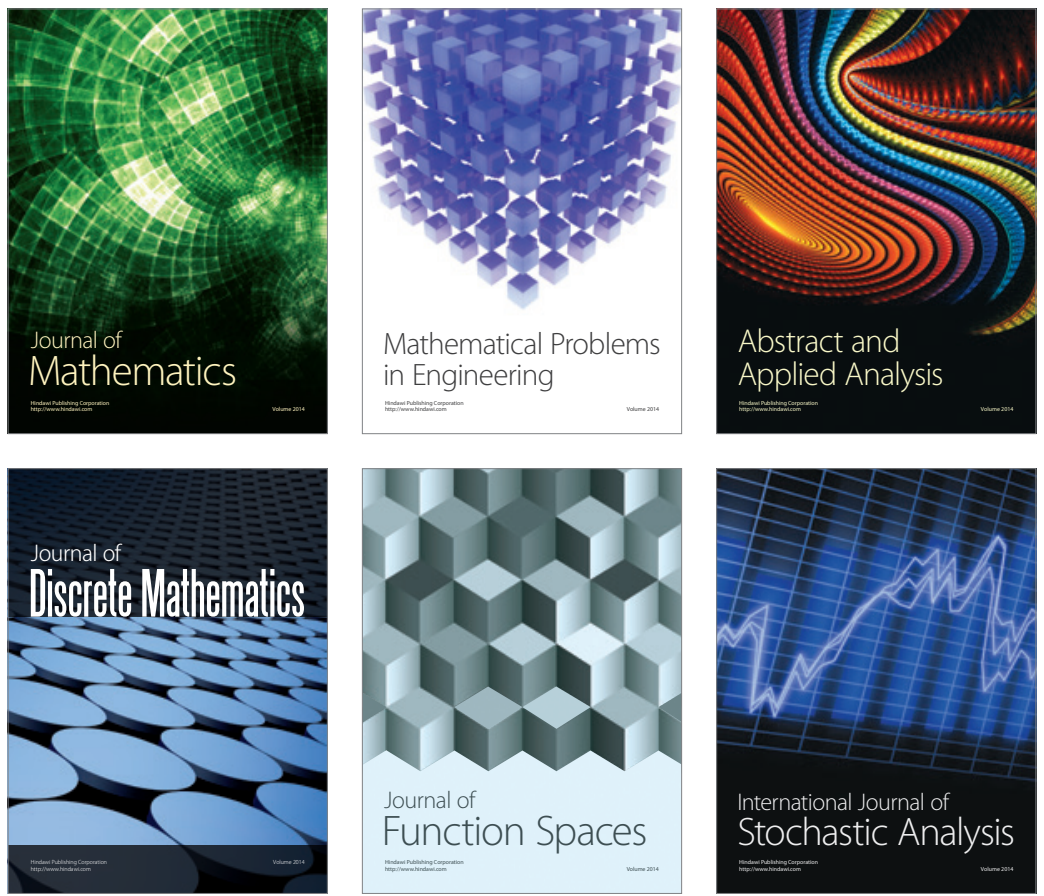

Journal of

Function Spaces

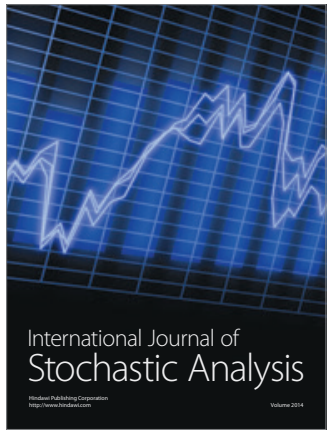

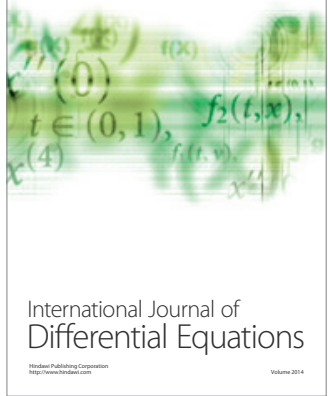
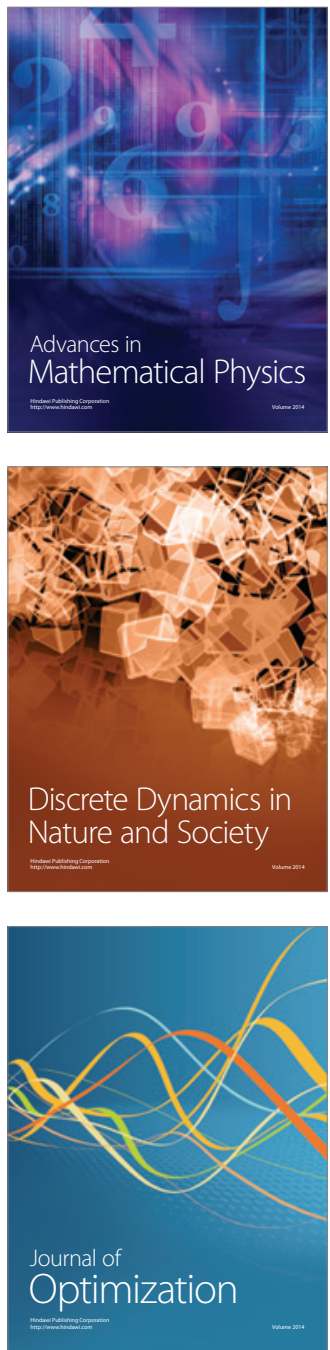